\title{
Synchrotron-based nano-XANES reveals intracellular heterogeneity of iron species in magnetotactic bacteria
}

\author{
Daniel M. Chevrier, ${ }^{1,2}$ Elisa Cerdá-Doñate, ${ }^{2}$ Yeseul Park, ${ }^{1}$ Fernando Cacho-Nerin, ${ }^{3}$ Miguel \\ Gomez-Gonzalez, ${ }^{3}$ René Uebe, ${ }^{4}$ Damien Faivre ${ }^{1,2^{*}}$ \\ ${ }^{1}$ Aix-Marseille Université, CNRS, CEA, BIAM, 13108 Saint-Paul-lez-Durance, France. \\ ${ }^{2}$ Max Planck Institute of Colloids and Interfaces, Department of Biomaterials, 14476 Potsdam, \\ Germany. \\ ${ }^{3}$ Diamond Light Source, Diamond House, Harwell Campus, Chilton, Didcot, OX11 0DE, U.K. \\ ${ }^{4}$ University of Bayreuth, Department of Microbiology, 95440 Bayreuth, Germany. \\ * Correspondence: Damien.faivre@cea.fr
}

Magnetotactic bacteria (MTB) sequester iron from the environment to biomineralize magnetite or greigite nanoparticles in magnetosome organelles, though the necessity of intracellular iron storage for the formation process is still in question. Understanding the role of iron storage would make clear the contribution of MTB in geochemical iron cycling and its pertinence to highly controlled biosynthesis of application-relevant magnetic nanoparticles. This report demonstrates how scanning X-ray fluorescence microscopy (SXFM) and nanoscale X-ray absorption near-edge structure (nano-XANES) can spatially and chemically identify intracellular iron species at the single-cell level, creating an opportunity to examine the role of iron storage in magnetite biomineralization. Fe K-edge nano-XANES measurements of Magnetospirillum gryphiswaldense in varied iron media conditions and iron storage capacity revealed intracellular iron heterogeneities through a distinction between formed magnetosomes and intracellular iron material. This work highlights the potential of nano-XANES in providing an experimental advantage in the multidisciplinary field of biomineralization.

\section{Introduction}

Recent breakthroughs in X-ray microscopy have enabled new means to investigate the structure and composition of materials ranging from energy-storage materials to individual cells to nanomaterials (1-5). In particular, X-ray nanoprobe beamlines at synchrotron facilities provide sub-100 $\mathrm{nm}$ beam sizes in the hard X-ray energy regime (i.e., $5-30 \mathrm{keV}$ ) that enable investigations of the nanoscopic composition, structure and organization of inorganic constituents in heterogeneous environments through an element-specific perspective (6-10). The advantage of hard X-ray nanoprobes is the combination of imaging and spectroscopy, where each pixel or position on the sample contains spectral information at the nanoscale (e.g., X-ray fluorescence (XRF), X-ray absorption spectroscopy (XAS), X-ray diffraction or scattering). Scanning X-ray fluorescence microscopy (SXFM) combined with such spectroscopy techniques has been highly advantageous for studying biological samples, revealing novel information on the intracellular biochemistry and role of metals in cells (11). Such X-ray nanoprobe advancements have investigated intracellular elemental composition and structure of algae $(8,12,13)$, bacteria (4), plants (14), and even small animals (15). Considering these milestones in nanoscale X-ray imaging of organisms, few studies have employed these approaches to address biological questions directly. 
Microorganism-controlled biomineralization represents one of the most sophisticated processes that produces inorganic nanomaterials. Magnetotactic bacteria (MTB) exemplarily synthesize single-domain magnetic nanoparticles composed of magnetite $\left(\mathrm{Fe}_{3} \mathrm{O}_{4}\right)$ or greigite $\left(\mathrm{Fe}_{3} \mathrm{~S}_{4}\right)$ within organelles known as magnetosomes. Magnetosomes are formed by a highly-controlled biological mechanism, where the number, morphology and arrangement of magnetic nanoparticles are species-specific $(16,17)$. The mechanism behind magnetite biomineralization in MTB is still unclear, with much left to understand in terms of iron uptake, iron storage, nanocrystal nucleation and growth (18). X-ray absorption fine structure (XAFS), Mössbauer spectroscopy and electron microscopy have been invaluable techniques to reveal initial insights into the biosynthesis of magnetite. Together, they have identified several iron oxide phases and iron precursors potentially involved in the formation mechanism (e.g., ferrihydrite, high-spin $\mathrm{Fe}^{2+}$ complexes, ferritin proteins, hematite, phosphate-rich ferric hydroxide) (19-22). More recently and relevant to the geochemical influence MTB have on iron cycling, studies have identified a large fraction of intracellular iron, at least partly composed of ferrous species, that is not incorporated into magnetosomes (23-25). The implications and localization of this stored iron are under question: whether it is pertinent to magnetite formation and if it is mainly found within magnetosome membranes $(24,26)$, contained by ferritin proteins $(27)$, or a more labile source in the cytoplasm $(24,28)$.

With these uncertainties in the forefront, X-ray spectromicroscopy techniques could lead to a clearer understanding of magnetosome formation by discerning iron species in distinct intracellular regions (e.g., membrane, cytoplasm, magnetosome chain) thereby avoiding convoluted chemicalstructural information from bulk measurements. Scanning transmission X-ray microscopy (STXM), which operates in the soft X-ray regime, has been employed recently to follow magnetosome formation and organization on the single-cell level, with additional information on the changing valence of iron and the evolution of magnetic properties using X-ray magnetic circular dichroism $(29,30)$. However, when probing the Fe L2,3-edge (700-730 eV) with STXM the short core-hole lifetime limits the collection of local structural information via X-ray backscattering processes. Higher energy Fe K-edge XAFS (7100-7700 eV) would be advantageous in this regard, especially for defining unknown iron species (e.g., amorphous precursors, subnanometer particles). Furthermore, STXM is a bright-field technique subject to a higher level of noise - transmission detection of photons not absorbed by sample - whereas SXFM is more sensitive for element-specific detection being a dark-field technique - directly measuring X-ray fluorescence events.

Herein, we demonstrate how hard X-ray microscopy can be employed as a new approach to probe magnetosome formation at the single-cell level via nano-XRF mapping and nano-XANES analysis. We measured wild-type Magnetospirillum gryphiswaldense MSR-1 bacteria and a genetic variant lacking ferritin proteins under varied iron concentrations and at different stages of magnetosome formation. Dark-field differential phase contrast X-ray imaging was first coupled with nano-XRF mapping to reconstruct both organic and inorganic components of bacterial cells to discern intracellular regions. Through principal components and cluster analyses of Fe K-edge nano-XANES data, we differentiated magnetosome chain structures from other intracellular iron species and determined their relative amounts. Linear combination fitting further identified the iron speciation in each distinct intracellular regions leading to a better understanding of iron storage under varied growth conditions and at early stages of biomineralization. 


\section{Results}

\section{Scanning X-ray fluorescence imaging of magnetotactic bacteria}

Individual M. gryphiswaldense cells were measured with scanning X-ray fluorescence microscopy (SXFM) using the X-ray nanoprobe beamline (I-14) at Diamond Light Source (10) and then with transmission electron microscopy (TEM) to confirm relative location of magnetosomes and cell membrane. Figure 1 displays bacteria characterized with both microscopy techniques (TEM measurements proceeded SXFM), presented in the same orientation. With an X-ray beam size of $\sim 50-60 \mathrm{~nm}$, approaching that of the fully formed magnetosomes in Fig. 1A (magnetosome diameter $=42 \pm 7 \mathrm{~nm}(\mathrm{n}=24)$, diameter range $=25-56 \mathrm{~nm})$, the Fe Ka XRF signal (Fig. 1B) displays the magnetosome chain configuration as seen from the TEM image (see Fig. S1 for XRF spectrum example). Even for smaller magnetosome particles, such as those for M. gryphiswaldense grown under iron-limited conditions in Fig. 1F (magnetosome diameter $=24 \pm 8 \mathrm{~nm}(\mathrm{n}=27)$, diameter range $=10-37 \mathrm{~nm}$ ), the Fe Ka XRF signal (Fig. 1G) still reflects the distribution of magnetosomes and orientation of the magnetosome chain (Table S1 lists the samples studied herein and their respective conditions). We also used X-rays scattered by the bacterium to produce differential phase contrast (DPC) images. Combining XRF and DPC signals produces a composite map that replicates the image seen from TEM (see Fig $1 \mathrm{~A} / \mathrm{E}$ and $1 \mathrm{~F} / \mathrm{J}$ ), enabling assessment of iron species and their intracellular location from SXFM data alone.

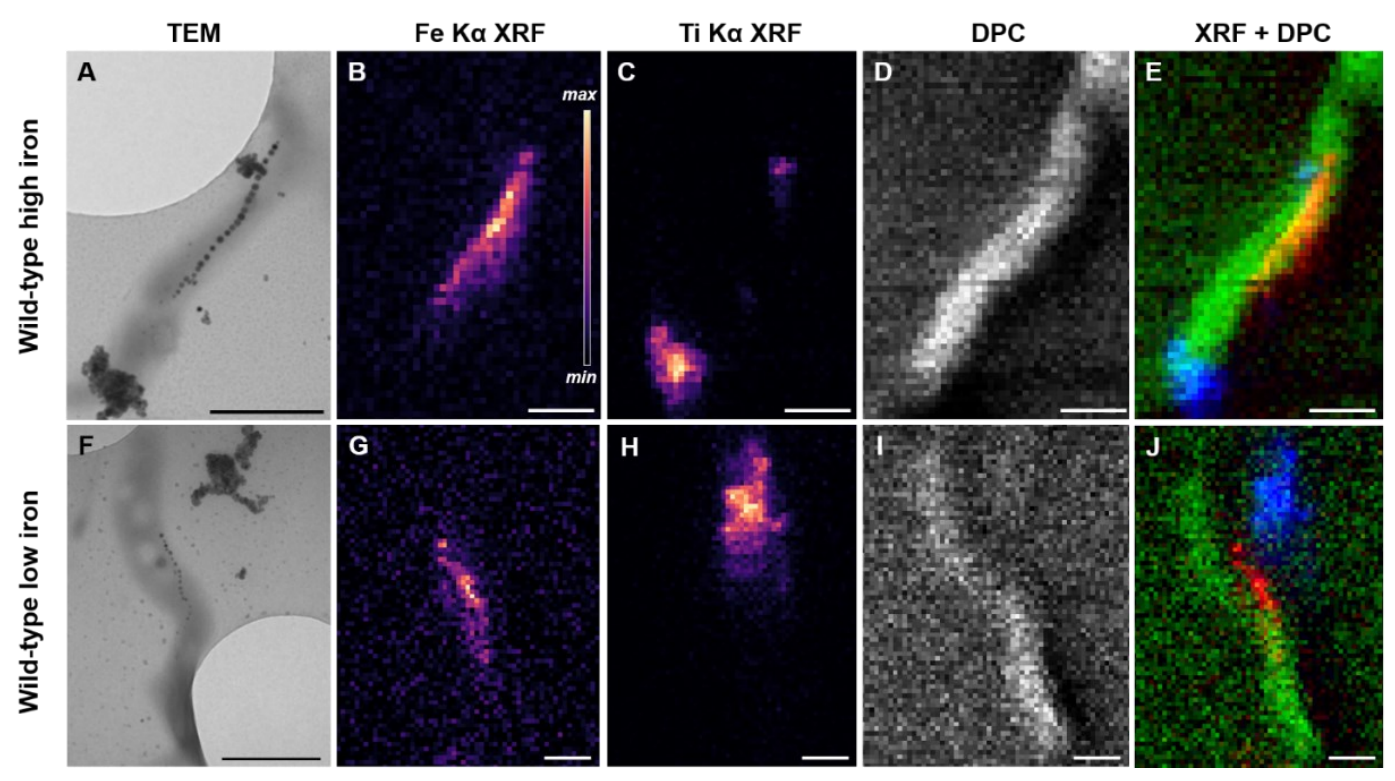

Fig. 1. Electron and X-ray fluorescence imaging of magnetotactic bacteria. Individual magnetotactic bacteria (wild-type $M$. gryphiswaldense) grown under standard conditions (first row, "WT high iron") and under iron-limited conditions (second row, "WT low iron") measured with transmission electron microscopy (TEM) (A and F) and scanning X-ray fluorescence microscopy (SXFM) (B-E and G-J). X-ray scattering was collected in transmission to produce differential phase contrast (DPC) maps to image cellular membrane material (D and I). Composite maps of Fe K $\alpha$ (red), Ti K $\alpha$ (blue) and DPC (green) are shown in E and J. Incident X-ray energy was $9 \mathrm{keV}$ with $\sim 50-60 \mathrm{~nm}$ X-ray beam diameter step size of $50 \mathrm{~nm}$. Scale bars are $1 \mu \mathrm{m}$ for TEM images and $500 \mathrm{~nm}$ for XRF and DPC maps. 
Though the spatial resolution of SXFM is unable to match that of TEM, this hard X-ray approach enables collection of Fe K-edge XAFS over the entire cell region, offering a new spectral vantage of chemical speciation at the single-cell level for MTB. A stack of XRF maps collected at varied energies across the Fe K-edge (i.e., 151 maps for 151 energy points) were combined and aligned to produce a stack or 2D XAFS data where each pixel translates to an XAFS spectrum. The XAFS data was truncated to show only the near-edge region due to weak post-edge oscillations, therefore the data is further referred to as nano-X-ray absorption near-edge structure (nano-XANES) (15, 31). To improve the tracking and alignment between each map during measurement and postprocessing, we deposited $\mathrm{TiO}_{2}$ nanoparticles onto the substrate to provide a reference point for the stack alignment procedure (see Fig. $1 \mathrm{~A} / \mathrm{C}$ and $1 \mathrm{~F} / \mathrm{H}$ ). The analysis of nano-XANES of four different samples in various states of biomineralization is presented below.

\section{Nano-XANES analysis of magnetosome formation}

We first consider the nano-XANES dataset for wild-type (WT) M. gryphiswaldense grown under standard conditions, where $50 \mu \mathrm{M}$ Fe(III)-citrate is added to ensure complete magnetosome formation (sample referred to as "WT high iron"). The Fe K-edge XANES signal from the entire cell region is presented in Fig. 2 with some iron oxide references for comparison. A positive shift in absorption edge energy is observed for WT high iron compared to magnetite $\left(\Delta E=E_{0}-E_{\mathrm{mag}}\right)$ of $+1.0 \mathrm{eV}$. Also found is broadening of the white-line (most intense absorption feature between $7130-7135 \mathrm{eV}$ ) and the near-edge feature around $7145 \mathrm{eV}$. The presence of ferric material is further confirmed with linear combination fitting (LCF) that yielded $60 \%$ magnetite $\left(\mathrm{Fe}_{3} \mathrm{O}_{4}\right), 33 \%$ hematite $\left(\mathrm{Fe}_{2} \mathrm{O}_{3}\right)$ and $7 \%$ goethite $(\mathrm{FeO}(\mathrm{OH}))\left(\mathrm{R}\right.$-factor $=0.0011$, ferrihydrite $\left(\mathrm{Fe}_{2} \mathrm{O}_{3} \cdot 0.5 \mathrm{H}_{2} \mathrm{O}\right)$, ferric chloride $\left(\mathrm{FeCl}_{3}\right)$ and ferrous chloride $\left(\mathrm{FeCl}_{2}\right)$ were also included as references in combinatorial fitting). LCF fitting results are summarized in Table S2 with XANES fits in Fig. S2-5. The high amount of ferric material could originate from ferric iron storage in ferritin proteins plus ferrous iron originally stored in the cell would have oxidized from sample preparation and ambient measurement conditions. We caution that herein LCF results help interpret the relative composition of intracellular iron species and to discern these from magnetosome particles but should not be taken directly for the actual composition. This is due to the XAFS measurement of nanostructured and amorphous materials that yield XANES spectra which do not resemble bulklike materials and thus may vary in near-edge features (e.g., spectral broadening, energy shift) (32, $33)$.

We utilized nano-XANES data to elucidate the location of iron species in the cell distinct from magnetite nanoparticles, where each pixel of the XRF map stack contains a XANES spectrum. To simplify the analysis of $>1000$ XANES spectra we used principal components and cluster analyses to objectively describe the variance in nano-XANES data, therefore aiding in the differentiation of distinct iron species. Principal components analysis (PCA) of nano-XANES data calculates eigenspectra and eigenimages from the map stack. Eigenspectra are an orthogonal set of spectra that, in linear combination, can describe any observed spectrum in the dataset. The eigenvalue associated with each eigenspectrum gives a typical weighting of that eigenspectrum in the entire dataset where eigenspectra with smaller eigenvalues represent less variations in the data. 
Examining the region indicated in Fig. 3A of WT high iron, PCA identified three components or three eigenimages (Fig. S6) that describe the variance in nano-XANES data. The second component shown in Fig. 3B (PC2, red region) accounts for most of the bacterium against the substrate background (where PC1 accounts for the bacterium and the substrate, Fig. S6). The third component (PC3) further distinguishes the magnetosome chain from intracellular iron species, confirmed by the agreement of magnetosome chain length and orientation in Fig. 1A and PC3 in Fig. 3B. Cluster analysis was then performed to group nano-XANES data according to spectral similarities into distinct intracellular regions, where the relative amount and location of distinct Fe K-edge XANES signals were inspected.

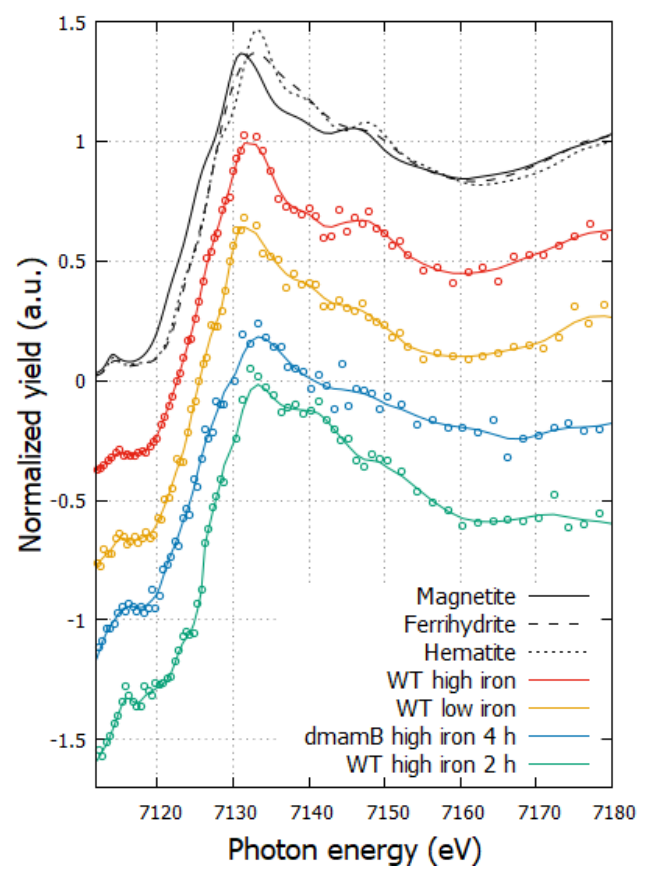

Fig. 2. XANES of individual magnetotactic bacteria. Fe K-edge XANES of reference materials and of full cell region for samples of M. gryphiswaldense: WT high iron, WT low iron, WT high iron $2 h$ and $\triangle m a m B$ high iron $4 h$.

Four cluster centers were sufficient to represent WT high iron nano-XANES (i.e., a fifth cluster did not further differentiate the XANES signal). The cluster centers map and corresponding XANES spectra for each cluster are presented in Fig. 3C and D, respectively. Cluster 1 encompasses the magnetosome chain as evidenced by accounting for $\sim 73 \%$ of the total XANES signal intensity, shown by $\mu(\mathrm{E})$ spectra retaining respective signal intensities for each cluster (Fig. $3 \mathrm{D}$, inset). The near-edge structure of cluster 1 resembles the magnetite reference similar to the full cell XANES, but again with a positive shift in absorption edge $\left(\Delta E_{0-\mathrm{mag}}=+1.1 \mathrm{eV}\right)$ and broader near-edge features, suggesting a mixture of components or partial oxidation to hematite or maghemite (Fe K-edge XANES for hematite and maghemite are similar (32)). LCF results are summarized in Fig. 3E, indicating a composition of 59\% magnetite, 33\% hematite and $8 \%$ goethite $(\mathrm{R}$-factor $=0.0011)$. Besides partial oxidation of magnetite, we expect iron-storage proteins such as ferritin to contribute to intracellular ferric oxide fitted components. 
LCF for cluster 2, which encircles cluster 1 and still within the intracellular region, yielded $16 \%$ hematite, $64 \%$ ferrihydrite, $7 \%$ ferric chloride and $13 \%$ ferrous chloride (R-factor $=0.0042$ ), and accounted for $\sim 13 \%$ of total XANES signal. We note that although ferric and ferrous chloride were fitted to the XANES spectrum of cluster 2 it does not directly indicate this particular species is present, rather it likely accounts for more ionic Fe(III) and Fe(II) species. Moreover, the components fitted reflect the iron material in the measured state, not the native state of the cell. Clusters 3 and 4, which represent the perimeter of the intracellular region and the substrate, respectively, have fewer distinguishable near-edge features than cluster 2 and combined account for less than $10 \%$ of the total XANES signal intensity. A larger portion of ferrihydrite is fitted due to broader XANES features. Cluster 4 is expectantly the weakest signal and gave a higher composition of iron chlorides which reflects the substrate background. The ferric oxide material associated with the magnetosome chain in cluster 1 indicated from LCF and measured absorption edge shift suggests magnetite nanoparticles could be partially oxidized from X-ray exposure and/or from iron storage species that have condensed around the magnetosome chain. The latter is suspected since PC2 (Fig. 3B) shows the total intracellular iron XANES signal to be concentrated around the magnetosome chain region and not dispersed throughout the entire cell.

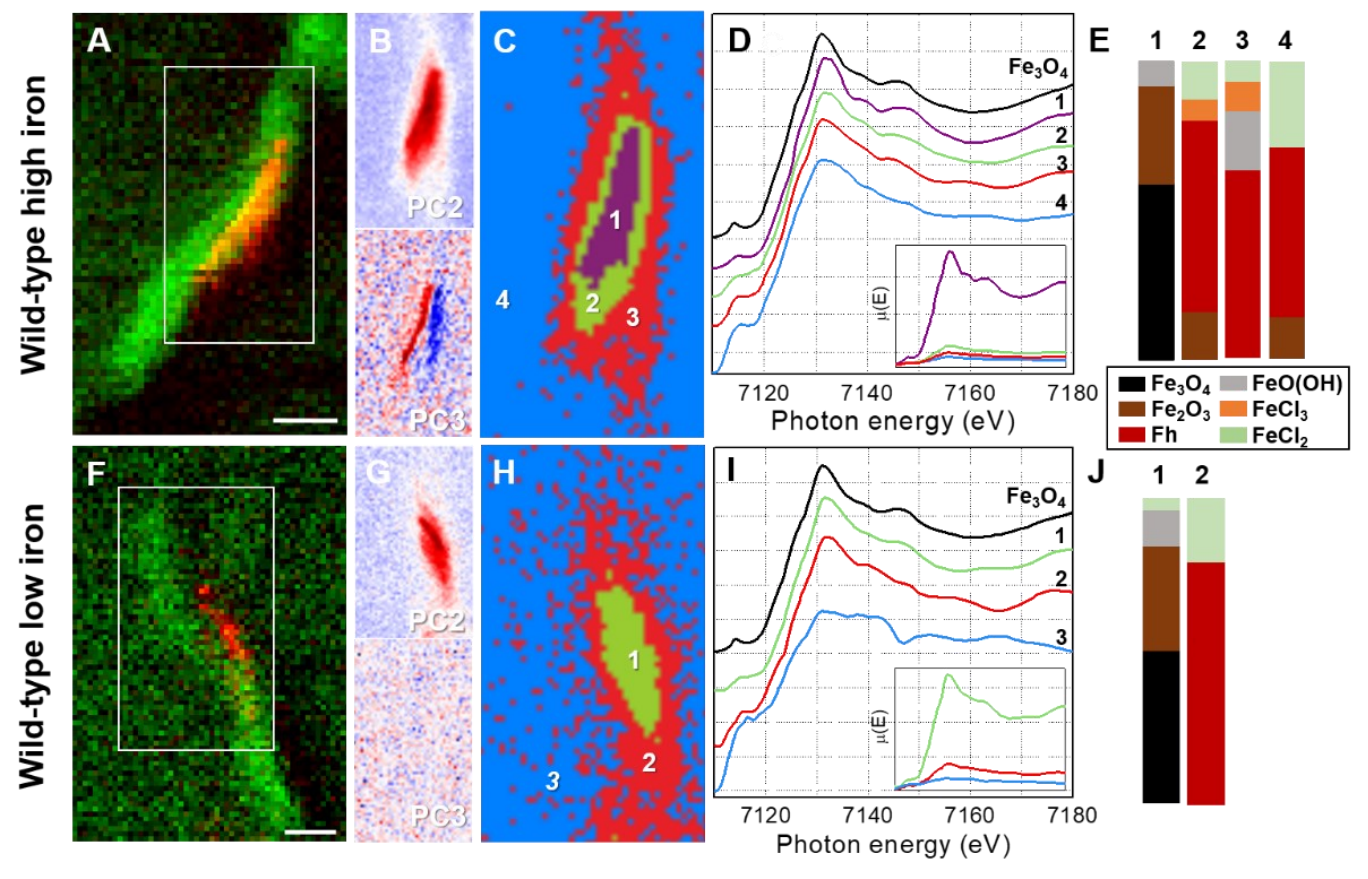

Fig 3. Nano-XANES data for magnetotactic bacteria with complete magnetosome chains. $W T$ high iron and WT low iron nano-XANES regions (A and F, white box), principal components analysis (PCA) maps (B and $\mathrm{G}$, red signal), maps of cluster centers ( $\mathrm{C}$ and $\mathrm{H}$ ), normalized offset Fe K-edge XANES spectra (D and I, inset with normalized spectra retaining edge jump values) and a summarized composite of linear combination fitting (LCF) results (E and J).

To address the intracellular iron content and potential magnetite oxidation from hard X-ray measurement, $M$. gryphiswaldense grown under low iron conditions to limit excess iron storage ("WT low iron", Fig. 1F) was inspected with nano-XANES. Examining the XANES spectrum for the entire cell in Fig. 2, again there are spectral similarities with magnetite and here with a more 
similar absorption edge position $\left(\Delta E_{0 \text {-mag }}=+0.4 \mathrm{eV}\right)$. LCF results for WT low iron gave $45 \%$ magnetite, $52 \%$ ferrihydrite and $3 \%$ ferrous chloride $(\mathrm{R}$-factor $=0.0017)$. In this case, the higher percentage of ferric material fitted may be caused by spectral broadening from the smaller magnetite nanoparticles (see Fig. 1F), which is also evidenced by the weaker near-edge feature at $7145 \mathrm{eV}$.

Different from WT high iron, a third PCA component did not account for the magnetosome chain in WT low iron (Fig. 3G), where this eigenimage shows random variations of the signal from pixelto-pixel. Cluster analysis with three cluster centers represented the concentric diversity of nanoXANES data outwards from the magnetosome chain, shown in Fig. 3H and I. Cluster 1 accounts for most of the region depicted in PC2 with $\sim 75 \%$ of the total signal intensity (inset Fig. 3I). Although the absorption edge energy shift is closer to magnetite $(+0.2 \mathrm{eV})$ than cluster 1 for $W T$ high iron, LCF results still show 50\% magnetite, 34\% hematite, $12 \%$ goethite and $4 \%$ ferrous chloride $(\mathrm{R}$-factor $=0.0016)$ indicating the presence of ferric oxide material in the magnetosome chain region. Cluster 2 shows similar absorption edge position to cluster 1 and near-edge features that are less defined due to the lower signal intensity ( $17 \%$ of total signal). This region is comparable to cluster 2 for WT high iron in terms of LCF results and absorption edge shift (see Table S2). The last cluster again represents the substrate region, but the XANES signal is too weak to characterize.

Similar compositions of magnetite and ferric oxides were found from the cluster analysis and LCF results of the magnetosome region for WT high iron and WT low iron (i.e., clusters 1 and 2). However, it is still not clear from WT low iron if the spectral broadening (evidenced by $\Delta E_{0 \text {-mag) }}$ from smaller nanoparticles enables fitting of ferric oxide references or if, in fact, it is caused by intracellular iron-storage. Therefore, intracellular iron storage was further limited by using a ferritin-deficient genetic variant of $M$. gryphiswaldense to suppress additional iron species in the cell and assess the oxidation of magnetite. Then, a comparison between early stage biomineralization of the ferritin-deficient variant and WT M. gryphiswaldense was made to discern nascent magnetosome particles from intracellular iron storage material.

\section{Early stage magnetosome formation}

Two additional M. gryphiswaldense samples - a genetic variant ( $\Delta$ mamB $\Delta$ dps/bfr12:: $\mathrm{P}_{\text {lac }}$-mamB, see Materials and Methods) and WT - were extracted from culture media a few hours after initiating magnetosome formation via different mechanisms. The genetic variant (referred to as $\triangle m a m B$ for short) is unable to form magnetosome membranes due to lack of the gene encoding for the MamB protein, which contributes to the structure of encapsulating membranes (34). $\triangle m a m B$ also lacks iron-storage proteins (e.g., ferritin family proteins) that act as iron reservoirs. This variant was designed so that magnetosome formation can be triggered via IPTG, a gene expression inducer (see Materials and Methods), to activate the mamB gene and resume magnetosome formation. The TEM image, Fe K $\alpha$ XRF map and DPC-XRF composite map are shown in Fig. 4A-C for a cell that was extracted from culture after $4 \mathrm{~h}$ post IPTG-induction (sample referred to as " $\triangle m a m B$ high iron $4 \mathrm{~h}$ "). More discrete Fe Ka XRF signals are observed compared to WT samples (see Fig. 4B and F) from the few magnetosomes (magnetosome diameter $=21 \pm 8$ $\mathrm{nm}(\mathrm{n}=5)$, diameter range $=11-30 \mathrm{~nm})$ and lack of ferritin material. 
Full cell XANES of $\triangle m a m B$ high iron $4 h$ in Fig. 2 and Fe Ka XRF map in Fig. 4B demonstrate that suppression of ferritin proteins greatly reduces intracellular iron during early biomineralization considerably. LCF fitting results for the full cell show $89 \%$ magnetite and $11 \%$

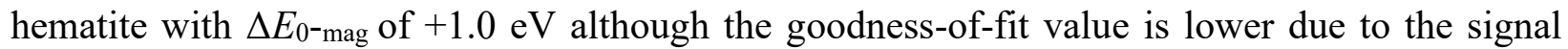
intensity of few nascent magnetosomes. Similar to WT low iron, PCA shows two components describe the variance in the data (Fig. 4D and S6) with the second eigenimage being discrete magnetosome particles. Likewise, three cluster centers were used to describe nano-XANES data (Fig. S7). LCF results for cluster 1 gave a high magnetite composition while cluster 2 resembles the intracellular composition found for $W T$ samples but with hematite instead of ferrihydrite. By avoiding interference from intracellular iron storage of ferritins, $\triangle \mathrm{mamB}$ high iron $4 \mathrm{~h}$ better infers the relative oxidation of magnetite nanoparticles expected from the hard X-ray measurement.
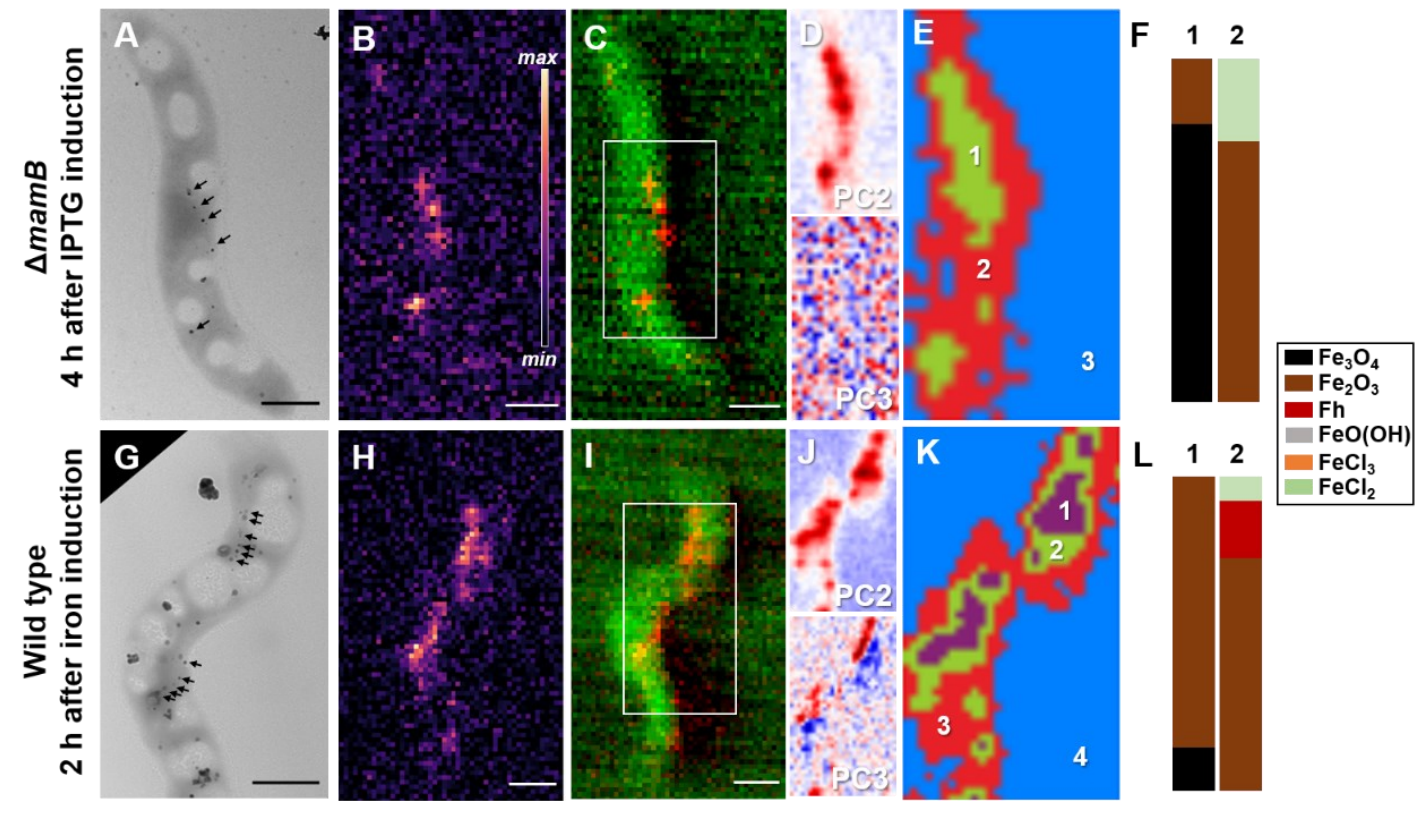

Fig. 4. TEM, SXRF and nano-XANES of magnetotactic bacteria in early stage magnetosome formation. M. gryphiswaldense $\triangle m a m B$ high iron $4 h$ (first row) and WT high iron $2 h$ (second row) at early stage biomineralization. TEM (A,G), Fe Ka XRF maps (B,H) and composite maps of Fe Ka XRF (red) and DPC (green) (C,I). Principal component maps for Fe K-edge nanoXANES $(\mathrm{D}, \mathrm{J})$, cluster maps $(\mathrm{E}, \mathrm{K})$ of indicated regions in composite maps and summarized linear combination fitting results $(\mathrm{F}, \mathrm{L})$. Incident X-ray energy $9 \mathrm{keV}$ for XRF and DPC maps. X-ray map pixel size $50 \mathrm{~nm}$. Scale bars $500 \mathrm{~nm}$.

The second early biomineralization sample was initially cultured under very low levels of iron to suppress magnetosome formation, introduced into iron-rich media to initiate biomineralization and then removed after $2 \mathrm{~h}$ (sample referred to as "WT high iron $2 \mathrm{~h}$ "). Fig. 4G-I displays TEM, Fe Ka $\mathrm{XRF}$ and the DPC-XRF composite map for WT high iron $2 h$, respectively. Compared to WT high iron and WT low iron, the Fe Ka XRF signal is dispersed throughout most of the cell with weaker signal intensity due to nascent magnetosomes (magnetosome diameter $=21 \pm 7 \mathrm{~nm}(\mathrm{n}=9)$, 
diameter range $=11-33 \mathrm{~nm}$ ). Despite this, two segments of forming magnetosome chain are still apparent in both TEM (indicated by arrows in Fig. 4G) and XRF data (Fig. 4H).

The full cell Fe K-edge XANES for WT high iron $2 h$ has a more pronounced near-edge feature following the white-line around $7140 \mathrm{eV}$, which could be attributed to more disordered ferric species, as observed for amorphous ferric iron from Dps-like proteins or phosphate-rich ferric hydroxides $(20,21)$. The full cell LCF result indicates $14 \%$ magnetite and $86 \%$ hematite with $\Delta E_{0^{-}}$ mag being $+1.6 \mathrm{eV}(\mathrm{R}$-factor $=0.0264)$, which suggests a high proportion of intracellular iron species distinct from magnetite are found in the cell at this early stage. As seen in Fig. 4J, the PC2 eigenimage presents most of the intracellular iron and PC3 accounts for the two developing magnetosome chain segments (see Fig. 4G indicated particles) similar to PC3 for WT high iron (Fig. 3B).

Four cluster centers were used to separate intracellular XANES signals as shown in Fig. 4K (cluster spectra shown in Fig. S7). Cluster 1 accounts for the magnetosome chain regions and cluster 2 for the intracellular region proximal to magnetosomes. The LCF result for cluster 1 yielded a composition similar to the full cell having only $14 \%$ magnetite and with a large $\Delta E_{0}$-mag of +1.8 $\mathrm{eV}(\mathrm{R}$-factor $=0.0113)$. Cluster $2 \mathrm{LCF}$ further reveals that iron species are mainly hematite and ferrihydrite. Although the magnetosome chain component is identifiable from PCA and cluster analysis (similar to WT high iron), PC2 and cluster 2 again demonstrate how intracellular iron storage is found close to magnetosome chain regions.

\section{Discussion}

Shown in this work, the intracellular iron heterogeneity of individual magnetotactic bacteria can be inspected with a semi-automated treatment of Fe K-edge nano-XANES data to reveal singlecell level information on iron homeostasis related to magnetosome production. Early-stage biomineralization samples illustrate how individual nascent magnetosomes can be distinguished from iron storage species by PCA and cluster analysis. While partial oxidation of magnetite was expected due to long measurement times and ambient atmosphere, the measurement of $\triangle \mathrm{mamB}$ high iron $4 \mathrm{~h}$ under the similar conditions and scanning parameters yielded a higher percentage of magnetite phase compared to other samples, albeit for smaller nanoparticles (i.e., higher surface area to volume, therefore more sensitive detection of surface oxidation by XANES). Without interference from ferritin storage, the extent of magnetite oxidation was assessed from cluster 1 of $\triangle m a m B$ high iron $4 h$, which was higher than WT samples. This suggests a significant contribution of ferric species detected as ferrihydrite or hematite in WT samples is likely from iron storage or oxidation of intracellular ferrous species (e.g., $\mathrm{Fe}$ (II) in cytoplasm or membrane structures that was oxidized during sample preparation/measurement) and not only from oxidation of magnetite. Although it is difficult to elucidate the exact chemical nature of iron species due to their amorphous and nanostructured nature, plus mixing and drying effects, our series of samples points to ferritin proteins as the main source of iron storage in M. gryphiswaldense that persists from early biomineralization to complete magnetosome chain formation.

The presence of ferritin proteins is not a surprise but its persistence throughout biomineralization is, as it may have complicated previous interpretations of time-dependent magnetosome formation 
studies. From time-course XAS studies with bulk samples, the appearance of magnetite nanoparticles in MTB quickly outweighs the contribution of intracellular ferric and ferrous species in the X-ray absorption signal collected (20-22). Therefore, amorphous iron species were detected at early time points and then thought to feed or precede magnetite formation, though it may persist throughout the entire biomineralization process as shown in this work. Recent studies with ferritinsuppressed mutants suggest that another mechanism may be at work when these proteins are absent and that accordingly, they may not be essential for biomineralization $(27,34)$. This highlights the plasticity of biological processes with the bacteria making use of intracellular material (i.e., ferritin) when it is present but utilizing an alternative predeveloped pathway when it is absent. It is only with nano-spectomicroscopy approaches such as SXFM and nano-XANES that ferritin can be detected directly and at varied stages of biomineralization.

For WT samples, iron storage species appear to agglomerate around the magnetosome chain, potentially from ferritin material that condenses onto magnetosome particles during the sample drying process. This is corroborated by eigenimages of PC2 for WT samples which show intracellular iron species are mainly found around magnetosomes and not throughout the entire cell. This is particularly evident for WT high iron $2 h$ where intracellular iron is found segmented according to the two portions of developing magnetosome chain (Fig. 4A and D). Although less ideal for capturing native-state information of the cell, the drying effects from sample preparation could be concentrating intracellular iron around such regions, which would lead to a higher intensity XANES signal. Considering XANES signal intensity from intracellular cluster regions outside of the magnetosome chain (e.g., clusters 2 and 3, regions outside magnetosome chain but within the cell membrane), around $22 \%, 16 \%, 28 \%$, and $48 \%$ is intracellular iron besides magnetite for WT high iron, WT low iron, $\triangle$ mamB high iron $4 h$, and WT high iron $2 h$, respectively. This still excludes additional iron species that are located around magnetosomes in cluster 1 . In fact, recent studies have reported similar substantial intracellular iron content separate from magnetosomes (23-25). In separate studies, Berny et al. and Amor et al. found that only 30-50\% of total intracellular iron content from both fully-grown MSR-1 and Magnetospirillum magneticum (AMB-1) cultures was used to produce magnetite. Amor et al. went on to characterize intracellular iron speciation and subcellular localization using fluorescence assays with AMB-1. Furthermore, they determined magnetite had only accounted for $\sim 30 \%$ of intracellular iron. This was further confirmed by a delay in magnetite precipitation as iron internalization increases.

Investigating intracellular biomineralization with Fe K-edge nano-XANES sets a precedent for the level of combined chemical-imaging information that is now achievable on the single-cell level with hard X-ray nanoprobe measurements. We demonstrate how this hard X-ray spectromicroscopy approach can be valuable to assess biological questions related to metals in living systems. SXFM and nano-XANES bridges the gap between high spatial resolution single cell imaging (e.g., HR-TEM) and high spectral resolution bulk characterization (e.g., scanning XAFS). This combined approach has the potential to recover more information on a cell-to-cell basis from a single experiment by combining adequate imaging and spectral resolution, enabling opportunities to make new progress in understanding biomineralization processes. Further improvements in X-ray nanoprobe instrumentation and measurements are expected with the availability of more fourth-generation synchrotron radiation facilities, offering brighter X-ray beams and higher coherence. For future work on magnetite biomineralization in MTB, conducting 
SXFM and nano-XANES under cryogenic conditions will enable vitrified samples to be measured to preserve iron homeostasis and reduce beam-induced changes to the sample. Another advantage of employing hard X-ray spectromicroscopy to study biomineralization processes is the emerging capabilities of integrated microfluidic cell sample environments to examine dynamic processes such as iron uptake and sequestration in situ.

\section{Materials and Methods}

\section{Culture media conditions, genetic variants, and sample preparation}

Magnetospirillum gryphiswaldense (MSR-1) was grown under microaerobic conditions in septum stoppered glass tubes ( $1 \%$ oxygen) in modified flask standard medium (FSM) for cultured magnetotactic bacteria.(35) M. gryphiswaldense collected after at least $72 \mathrm{~h}$ of growth is referred to as "WT high iron". M. gryphiswaldense was also grown in modified low iron media conditions (no Fe(III)-citrate added to FSM), where samples collected after $72 \mathrm{~h}$ of growth are referred to as "WT low iron". For magnetosome induction experiments, M. gryphiswaldense was first grown in modified FSM medium with very low iron (no Fe(III)-citrate and no peptone) and under aerobic conditions for at least two passages. In the third passage, cultures were transferred at early logarithmic phase to FSM (high iron conditions) and under microaerobic conditions to induce magnetosome formation. An aliquot was removed after $2 \mathrm{~h}$ of exposure to iron-rich media and is referred to "WT high iron $2 \mathrm{~h}$ " sample. Alternative to this magnetosome induction method, a genetic variant was used to trigger magnetosome formation via genetic induction. The essential mamB gene was deleted within the M. gryphiswaldense ferritin genetic variant $\Delta d p s / b f r 12$ (27) using the plasmid pOR $\triangle \operatorname{mamB} B$.(36) This genetic variant was then complemented by site-specific, Tn7 transposon-mediated chromosomal integration of an isopropyl $\beta$-D-1-thiogalacto-pyranoside (IPTG) inducible $\mathrm{P}_{\text {lac }}$-mamB construct.(34) The resulting strain $\Delta$ mamB $\Delta$ dps/bfr $12: \because \mathrm{P}_{\text {lac }}$-mamB was grown under conditions similar to WT high iron with the exception that $2 \mathrm{mM}$ IPTG was added. A sample was removed $4 \mathrm{~h}$ after IPTG induction and referred to as " $\triangle$ mamB high iron 4 $h "$.

Samples of M. gryphiswaldense were taken as small aliquots from culture media, centrifuged to remove culture media and then washed with 0.01 M HEPES and 0.02 M EDTA buffer $(\mathrm{pH} 7)$ three times to remove excess metal ions. Finally, cells were washed once with Milli-Q water $\left(18.2 \Omega \mathrm{m}^{-}\right.$ ${ }^{1}$ ). $5 \mu \mathrm{L}$ of washed cells were deposited onto parafilm with a TEM grid (gold or copper metal with holey carbon film) placed on top of each drop (carbon side down) for at least $30 \mathrm{~min}$. Afterwards, the grids were submerged in Milli-Q water for $10 \mathrm{~s}$ three times and then dried on filter paper. $5 \mu \mathrm{L}$ of a 1/50000 diluted $\mathrm{TiO}_{2}$ nanoparticle solution $\left(\sim 150 \mathrm{~nm}\right.$ in diameter, $33 \mathrm{wt} \% \mathrm{TiO}_{2}$ in water, Sigma Aldrich) was added to each TEM grid to provide a measurable secondary XRF signal for tracking and alignment purposes for XANES mapping acquisition of single cells.

\section{Hard X-ray nanoprobe measurements}

TEM grids were mounted for measurement at the I-14 hard X-ray nanoprobe beamline (Diamond Light Source Ltd., Didcot, UK) using custom holders designed and supplied by the beamline (10). SXFM measurements were conducted under ambient conditions using an incident photon energy of $9 \mathrm{keV}$ for XRF mapping and a range of 7.0-7.3 keV for collection of Fe K-edge X-ray absorption near-edge structure (XANES) maps. The focused X-ray beam was 50-60 nm (FWHM) in diameter. $\mathrm{X}$-ray fluorescence emission (XRF) from the sample was collected in front of the sample using a four-element silicon drift detector (RaySpec, UK). A raster scanning step size of 50-100 nm was 
used with a dwell time of 30-60 ms to collect high resolution XRF maps. A photon-counting Merlin detector (Quantum Detectors, UK) was also used in transmission configuration to collect X-ray scattering for differential phase contrast (DPC) images.

\section{XAFS and Nano-XANES measurements}

Reference materials were prepared as pellets and used for qualitative comparison and quantitative analysis of XAFS data (purified magnetosomes, $\mathrm{Fe}_{3} \mathrm{O}_{4}, \mathrm{Fe}_{2} \mathrm{O}_{3}, 2$-line ferrihydrite, $\mathrm{FeCl}_{2}, \mathrm{FeCl}_{3}$ ). Enough iron oxide material was combined and mixed with cellulose (using pestle and mortar) to achieve one X-ray absorption length. XAFS measurements of references were collected in transmission mode at the I-14 nanoprobe using a photodiode detector. Nano-XANES mapping was performed by collecting a series of XRF maps across the Fe K-edge. To correct for drift during the measurement and to align the maps afterwards, the Ti K $\alpha$ XRF signal was used as a tracking and post-alignment feature. Presented XAFS spectra from nano-XANES mapping were smoothed using 3-point averaging (Figure 2 presents raw data and smoothed data for comparison).

\section{TEM analysis}

TEM images were collected using a Tecnai G2 BioTWIN (FEI Company) electron microscope equipped with a CCD camera (Megaview III, Olympus Soft imaging Solutions GmbH) using an accelerating voltage of $100 \mathrm{kV}$.

\section{Data and image analysis}

Image J (Fiji version) was used to analyze TEM images and conduct particle size measurements. Athena program from the Demeter package was employed to conduct XAFS data normalization, energy calibration and linear combination fitting (LCF).(37) A combinatorial approach was used for LCF analysis by testing all combinations of reference materials (magnetite, hematite, goethite, ferrihydrite, ferric chloride and ferrous chloride). The best fit was obtained by selecting the lowest number of references required to obtain a fit whose reduced- $\chi^{2}$ value was not improved by $10 \%$ with an additional reference. When fitting cluster center spectra, the magnetite reference was omitted when the cluster was outside the magnetosome chain region. $\operatorname{Dawn}(38)$ and $\operatorname{Mantis}(39)$ software were used to interpret collected XRF maps and nano-XANES dataset, with the latter used for principal components and cluster center analyses. Similarly, Python-based scripts created by I14 Nanoprobe group at Diamond were employed to perform normalization, alignment, and cluster analysis of nano-XANES data. Silhouette scores / error maps were used to determine the minimum number of components required to describe the nano-XANES dataset (limiting variance between cluster signals within its own group using the Cattell scree test, where the number of significant components is given by detecting an "elbow" feature in the eigenvalue plot).(40)

\section{Acknowledgments}

We would like to acknowledge the "Institut de Radioprotection et de Sûreté Nucléaire" (IRSN) at CEA Cadarache for access of TEM facilities, Dr. Christopher Lefèvre for conducting TEM measurements and Dr. Matthieu Amor for helpful and insightful comments on this work. D.M.C. acknowledges research funding through a European Union Marie-Skłodowska Curie Action International Fellowship (MSCA-IF Project 797431: BioNanoMagnets). D.M.C. and D.F. acknowledge support from the Max Planck Society. E.C.D. was supported by the IMPRS on Multiscale Bio-Systems. We would like to acknowledge Diamond Light Source for beamtime on 
Beamline I-14 under proposals MG23693-1 and MG23602-1. R.U. acknowledges funding received from Deutsche Forschungsgemeinschaft via grant UE200/1-1.

\section{References}

1. A. Sakdinawat, D. Attwood, Nanoscale X-ray imaging. Nat. Photonics. 4, 840-848 (2010).

2. D. A. Shapiro, Y. S. Yu, T. Tyliszczak, J. Cabana, R. Celestre, W. Chao, K. Kaznatcheev, A. L. D. Kilcoyne, F. Maia, S. Marchesini, Y. S. Meng, T. Warwick, L. L. Yang, H. A. Padmore, Chemical composition mapping with nanometre resolution by soft X-ray microscopy. Nat. Photonics. 8, 765-769 (2014).

3. J. Wang, Y. C. K. Chen-Wiegart, J. Wang, In operando tracking phase transformation evolution of lithium iron phosphate with hard X-ray microscopy. Nat. Commun. 5, 1-10 (2014).

4. T. W. Victor, L. M. Easthon, M. Ge, K. H. O’Toole, R. J. Smith, X. Huang, H. Yan, K. N. Allen, Y. S. Chu, L. M. Miller, X-ray Fluorescence Nanotomography of Single Bacteria with a Sub-15 nm Beam. Sci. Rep. 8, 13415 (2018).

5. C. Y. J. Hémonnot, S. Köster, Imaging of Biological Materials and Cells by X-ray Scattering and Diffraction. ACS Nano. 11, 8542-8559 (2017).

6. A. Pattammattel, R. Tappero, M. Ge, Y. S. Chu, X. Huang, Y. Gao, H. Yan, Highsensitivity nanoscale chemical imaging with hard x-ray nano-XANES. Sci. Adv. 6, 1-8 (2020).

7. K. M. Kemner, S. D. Kelly, B. Lai, J. Maser, E. J. O’Loughlin, D. Sholto-Douglas, Z. Cai, M. A. Schneegurt, C. F. Kulpa, K. H. Nealson, Elemental and redox analysis of single bacterial cells by X-ray microbeam analysis. Science (80-. ). 306, 686-687 (2004).

8. J. Deng, D. J. Vine, S. Chen, Y. S. G. Nashed, Q. Jin, N. W. Phillips, T. Peterka, R. Ross, S. Vogt, C. Jacobsen, Simultaneous cryo X-ray ptychographic and fluorescence microscopy of green algae. Proc. Natl. Acad. Sci. 112, 2314-2319 (2015).

9. R. P. Winarski, M. V. Holt, V. Rose, P. Fuesz, D. Carbaugh, C. Benson, D. Shu, D. Kline, G. Brian Stephenson, I. McNulty, J. Maser, A hard X-ray nanoprobe beamline for nanoscale microscopy. J. Synchrotron Radiat. 19, 1056-1060 (2012).

10. P. D. Quinn, L. Alianelli, M. Gomez-gonzalez, D. Mahoney, F. Cacho-nerin, A. Peach, J. E. Parker, The Hard X-ray Nanoprobe beamline at Diamond Light Source. J. Synchrotron Radiat. 28, 1-8 (2021).

11. D. J. Hare, E. J. New, M. D. De Jonge, G. McColl, Imaging metals in biology: Balancing sensitivity, selectivity and spatial resolution. Chem. Soc. Rev. 44, 5941-5958 (2015).

12. J. Deng, D. J. Vine, S. Chen, Q. Jin, Y. S. G. Nashed, T. Peterka, S. Vogt, C. Jacobsen, Xray ptychographic and fluorescence microscopy of frozen-hydrated cells using continuous scanning. Sci. Rep. 7, 445 (2017).

13. J. Deng, Y. H. Lo, M. Gallagher-jones, S. Chen, A. P. Jr, Q. Jin, Y. P. Hong, Y. S. G. Nashed, S. Vogt, J. Miao, C. Jacobsen, Correlative 3D x-ray fluorescence and ptychographic tomography of frozen-hydrated green algae. Sci. Adv. 4, eaau4548 (2018).

14. G. Vigani, S. Bohic, F. Faoro, B. Vekemans, L. Vincze, R. Terzano, Cellular Fractionation and Nanoscopic X-Ray Fluorescence Imaging Analyses Reveal Changes of Zinc Distribution in Leaf Cells of Iron-Deficient Plants. Front. Plant Sci. 9, 1112 (2018).

15. S. A. James, D. J. Hare, N. L. Jenkins, M. D. De Jonge, A. I. Bush, G. McColl, $\phi$ 
XANES : In vivo imaging of metal- protein coordination environments. Sci. Rep. 6, 20350 (2016).

16. D. Faivre, D. Schuler, Magnetotactic Bacteria and Magnetosomes. Chem. Rev. 108, 48754898 (2008).

17. R. Uebe, D. Schüler, Magnetosome biogenesis in magnetotactic bacteria. Nat. Rev. Microbiol. 14, 621-637 (2016).

18. M. Amor, F. P. Mathon, C. L. Monteil, V. Busigny, C. T. Lefevre, Iron-biomineralizing organelle in magnetotactic bacteria: function, synthesis and preservation in ancient rock samples. Environ. Microbiol. 22, 3611-3632 (2020).

19. D. Faivre, L. H. Böttger, B. F. Matzanke, D. Schüler, Intracellular magnetite biomineralization in bacteria proceeds by a distinct pathway involving membrane-bound ferritin and an iron(II) species. Angew. Chemie - Int. Ed. 46, 8495-8499 (2007).

20. J. Baumgartner, G. Morin, N. Menguy, T. Perez Gonzalez, M. Widdrat, J. Cosmidis, D. Faivre, Magnetotactic bacteria form magnetite from a phosphate-rich ferric hydroxide via nanometric ferric (oxyhydr)oxide intermediates. Proc. Natl. Acad. Sci. U. S. A. 110, 14883-8 (2013).

21. M. L. Fdez-gubieda, A. Muela, J. Alonso, A. Garci-Prieto, L. Olivi, R. FernandezPacheco, J. Manuel Barandiaran, Magnetite Biomineralization in Magnetospirillum gryphiswaldense : Time-resolved Magnetic and Structural Studies. ACS Nano. 7, $3297-$ 3305 (2013).

22. S. Staniland, B. Ward, A. Harrison, G. van der Laan, N. Telling, Rapid magnetosome formation shown by real-time x-ray magnetic circular dichroism. Proc. Natl. Acad. Sci. 104, 19524-19528 (2007).

23. M. Amor, V. Busigny, P. Louvat, M. Tharaud, A. Gelabert, P. Cartigny, J. Carlut, A. Isambert, M. Durand-Dubief, G. Ona-Nguema, E. Alphandéry, I. Chebbi, F. Guyot, Iron uptake and magnetite biomineralization in the magnetotactic bacterium Magnetospirillum magneticum strain AMB-1 : An iron isotope study. Geochim. Cosmochim. Acta. 232, 225243 (2018).

24. M. Amor, A. Ceballos, J. Wan, C. P. Simon, A. T. Aron, C. J. Chang, F. Hellman, A. Komeili, Magnetotactic Bacteria Accumulate a Large Pool of Iron Distinct from Their Magnetite Crystals. Appl. Environ. Microbiol. 86, e01278-20 (2020).

25. C. Berny, R. Le Fèvre, F. Guyot, K. Blondeau, C. Guizonne, E. Rousseau, N. Bayan, E. Alphandéry, A Method for Producing Highly Pure Magnetosomes in Large Quantity for Medical Applications Using Magnetospirillum gryphiswaldense MSR-1 Magnetotactic Bacteria Amplified in Minimal Growth Media. Front. Bioeng. Biotechnol. 8 (2020), doi:10.3389/fbioe.2020.00016.

26. J. Li, H. Zhang, N. Menguy, K. Benzerara, F. Wang, X. Lin, Zhibao Chen, Y. Pana, Single-Cell Resolution of Uncultured Magnetotactic Bacteria via Fluorescence-Coupled Electron Microscopy. Appl. Environ. Microbiol. 83, e00409-17 (2017).

27. R. Uebe, F. Ahrens, J. Stang, K. Jäger, L. H. Böttger, C. Schmidt, B. F. Matzanke, D. Schüler, Bacterioferritin of Magnetospirillum gryphiswaldense Is a Heterotetraeicosameric Complex Composed of Functionally Distinct Subunits but Is Not Involved in Magnetite Biomineralization. MBio. 10, e02795-18 (2019).

28. O. Kakhlon, Z. I. Cabantchik, The Labile Iron Pool: Characterization, Measurement, and Participation in Cellular Processes. Free Radic. Biol. Med. 33, 1037-1046 (2002).

29. X. Zhu, A. P. Hitchcock, D. A. Bazylinski, P. Denes, J. Joseph, U. Lins, S. Marchesini, 
H.-W. Shiu, T. Tyliszczak, D. A. Shapiro, Measuring spectroscopy and magnetism of extracted and intracellular magnetosomes using soft X-ray ptychography. Proc. Natl. Acad. Sci. U. S. A. 113, E8219-E8227 (2016).

30. L. Le Nagard, X. Zhu, H. Yuan, K. Benzerara, D. A. Bazylinski, C. Fradin, A. Besson, S. Swaraj, S. Stanescu, R. Belkhou, A. P. Hitchcock, Magnetite magnetosome biomineralization in Magnetospirillum magneticum strain AMB-1: A time course study. Chem. Geol. 530, 119348 (2019).

31. A. Pattammattel, R. Tappero, M. Ge, Y. S. Chu, X. Huang, Y. Gao, H. Yan, Highsensitivity nanoscale chemical imaging with hard x-ray nano-XANES. Sci. $A d v .6$, eabb3615 (2020).

32. C. Piquer, M. A. Laguna-Marco, A. G. Roca, R. Boada, C. Guglieri, J. Chaboy, Fe K-edge X-ray absorption spectroscopy study of nanosized nominal magnetite. J. Phys. Chem. C. 118, 1332-1346 (2014).

33. A. Kuzmin, J. Chaboy, EXAFS and XANES analysis of oxides at the nanoscale. IUCrJ. 1, 571-589 (2014).

34. O. Raschdorf, Y. Forstner, I. Kolinko, R. Uebe, J. M. Plitzko, D. Schüler, Genetic and Ultrastructural Analysis Reveals the Key Players and Initial Steps of Bacterial Magnetosome Membrane Biogenesis. PLoS Genet. 12, 1-23 (2016).

35. U. Heyen, D. Schüler, Growth and magnetosome formation by microaerophilic Magnetospirillum strains in an oxygen-controlled fermentor. Appl. Microbiol. Biotechnol. 61, 536-544 (2003).

36. R. Uebe, N. Keren-Khadmy, N. Zeytuni, E. Katzmann, Y. Navon, G. Davidov, J. M. Plitzko, D. Schuler, R. Zarivach, The dual role of MamB in magnetosome membrane assembly and magnetite biomineralization. Mol. Microbiol. 107, 542-557 (2018).

37. B. Ravel, M. Newville, ATHENA, ARTEMIS, HEPHAESTUS: data analysis for X-ray absorption spectroscopy using IFEFFIT. J. Synchrotron Radiat. 12, 537-541 (2005).

38. M. Basham, J. Filik, M. T. Wharmby, P. C. Y. Chang, B. El Kassaby, M. Gerring, J. Aishima, K. Levik, B. C. A. Pulford, I. Sikharulidze, D. Sneddon, M. Webber, S. S. Dhesi, A. W. Ashton, computer programs Data Analysis WorkbeNch ( DAWN ), 853-858 (2015).

39. M. Lerotic, R. Mak, S. Wirick, F. Meirer, C. Jacobsen, MANTiS: a program for the analysis of X-ray spectromicroscopy data. J. Synchrotron Radiat. 21, 1206-1212 (2014).

40. R. B. Cattell, The Scree Test For The Number Of Factors. Multivariate Behav. Res. 1, 245-276 (1966). 


\section{Supplementary Materials}

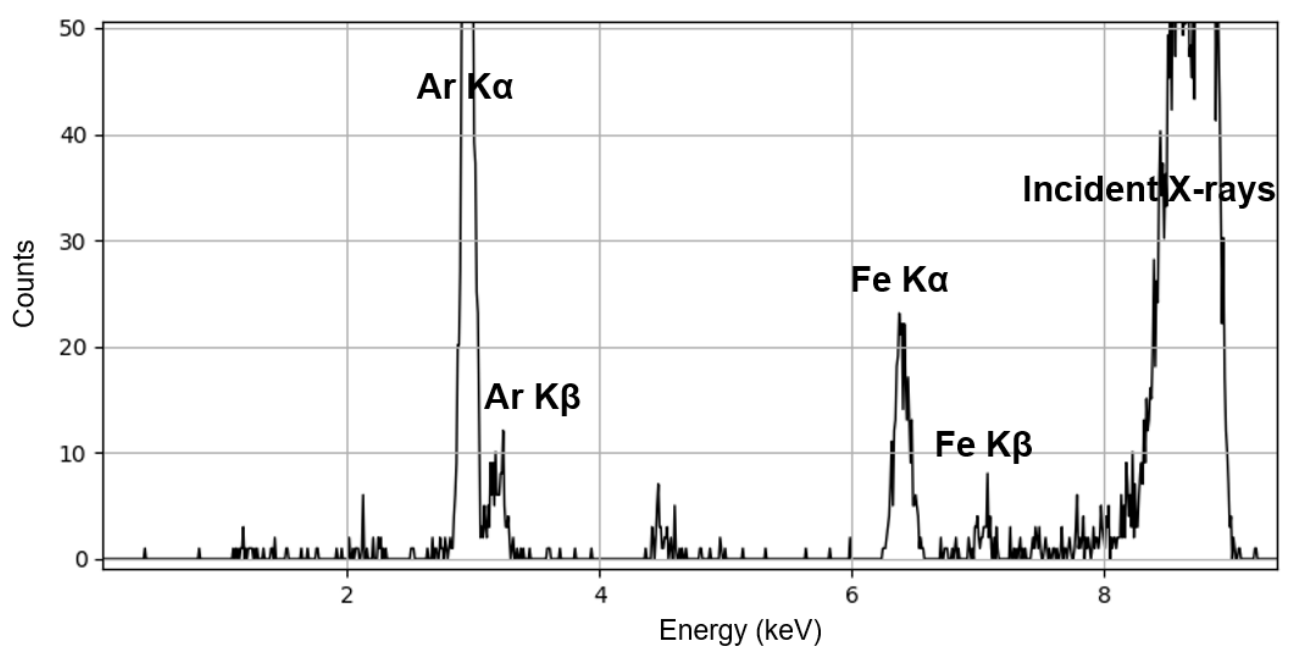

Fig S1. XRF spectrum from magnetosome chain region of MSR-1 WT high iron (Fig 1A). 

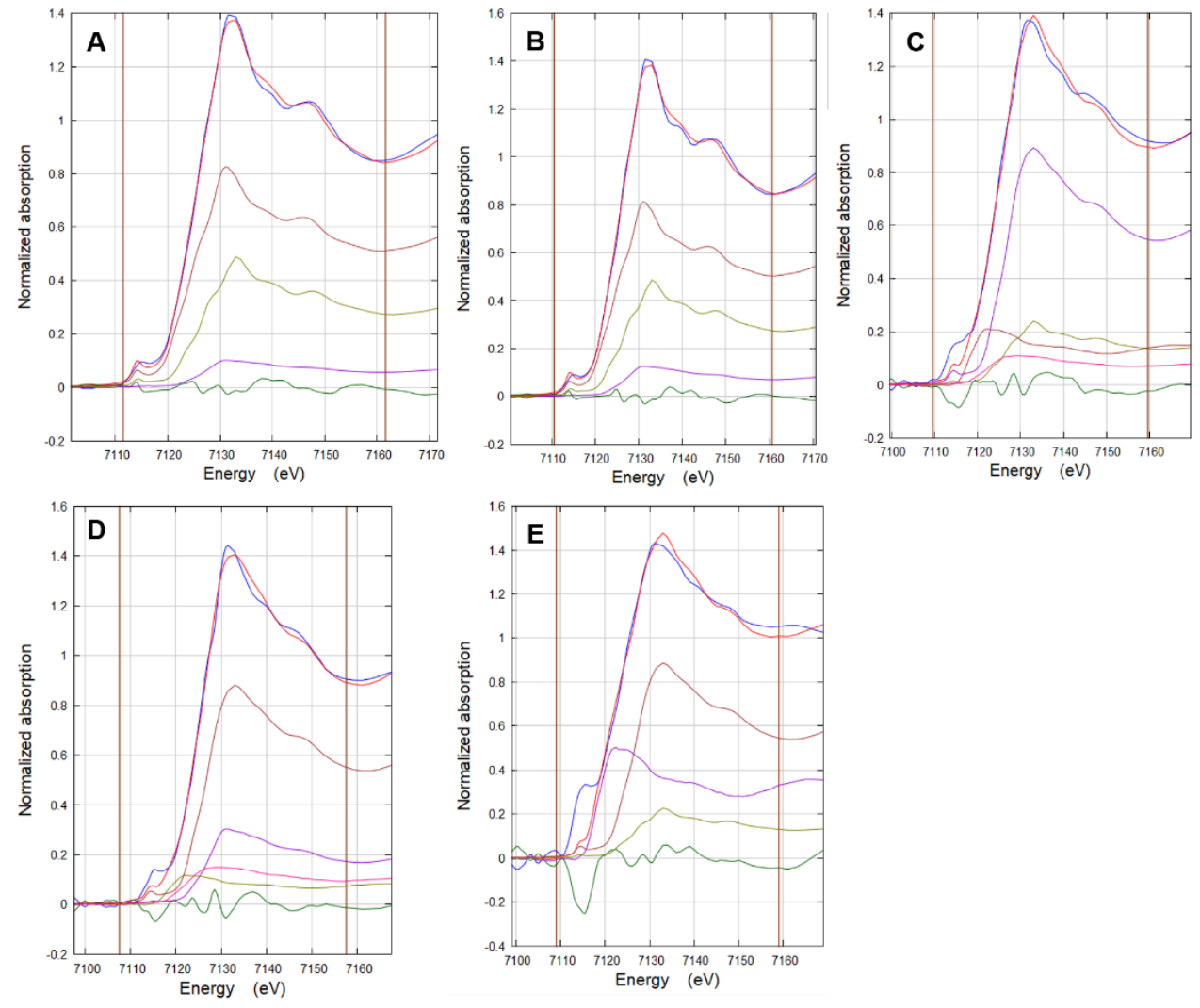

Fig S2. Linear combination fitting of WT high iron Fe K-edge XANES data for full cell region (A) and fitted cluster centers (cluster 1 (B), cluster 2 (C), cluster 3 (D) and cluster 4 (E)). Blue line - experimental data, red line - fit, green - residual from fit, vertical brown lines - fitting region. 

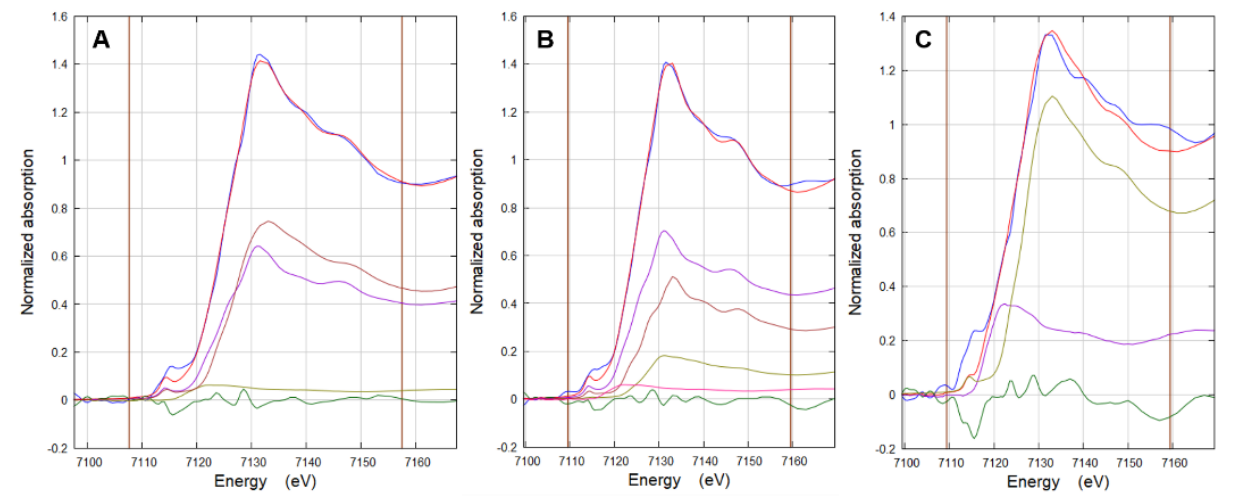

Fig S3. Linear combination fitting of WT low iron Fe K-edge XANES data for full cell region (A) and fitted cluster centers (cluster 1 (B) and cluster 2 (C)). Blue line - experimental data, red line - fit, green - residual from fit, vertical brown lines - fitting region. 

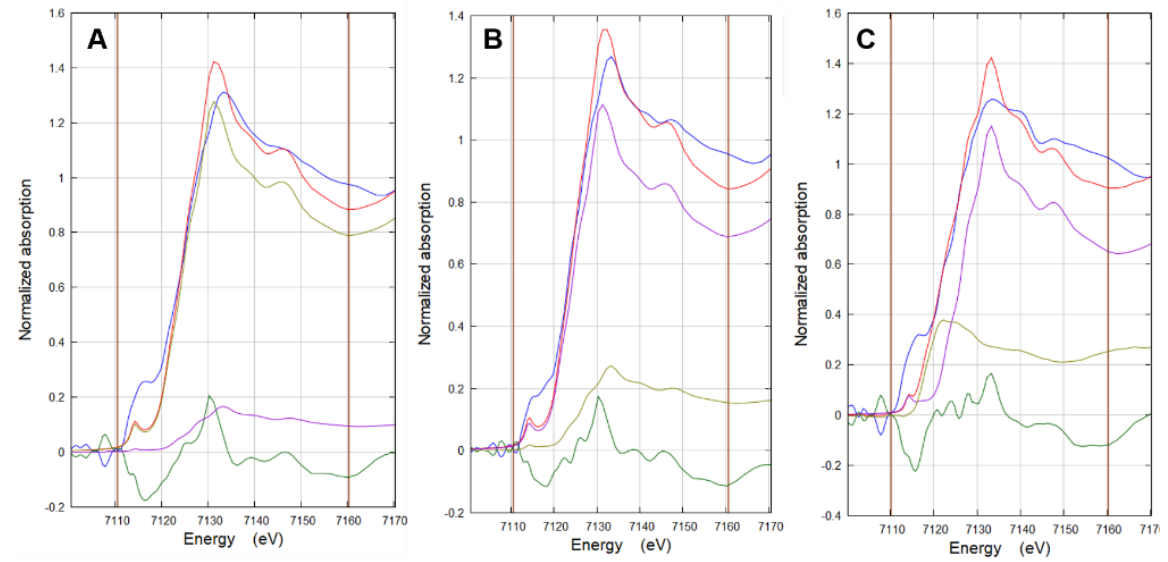

Fig S4. Linear combination fitting of $\triangle m a m B$ high iron $4 h$ Fe K-edge XANES data for full cell region (A) and fitted cluster centers (cluster 1 (B) and cluster 2 (C)). Blue line - experimental data, red line - fit, green - residual from fit, vertical brown lines - fitting region. 

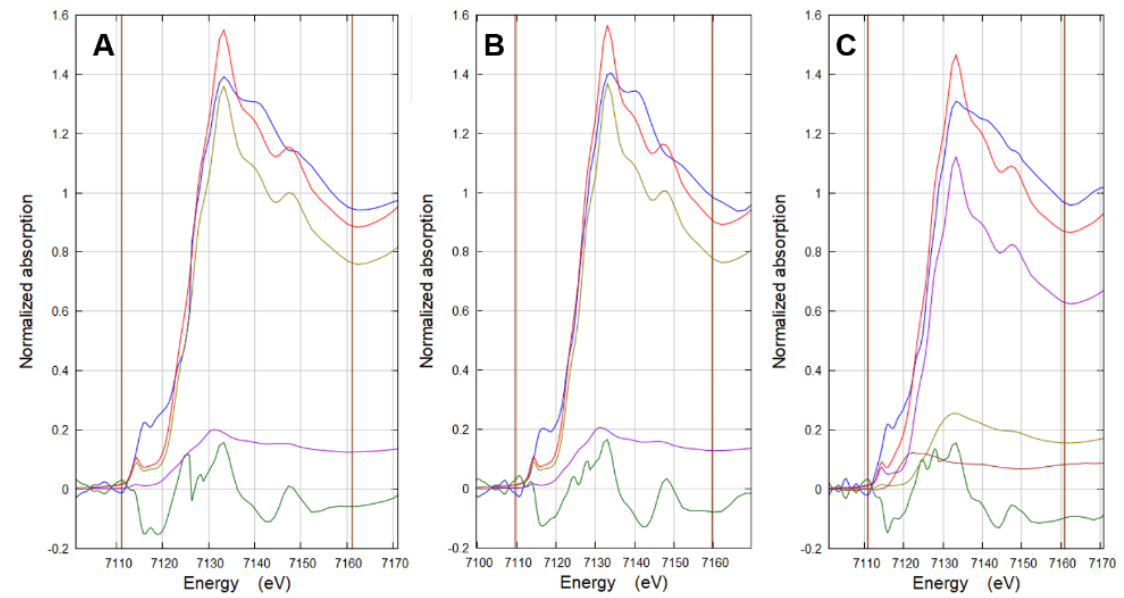

Fig S5. Linear combination fitting of WT high iron $2 h \mathrm{Fe}$ K-edge XANES data for full cell region (A) and fitted cluster centers (cluster 1 (B) and cluster 2 (C)). Blue line - experimental data, red line - fit, green - residual from fit, vertical brown lines - fitting region. 


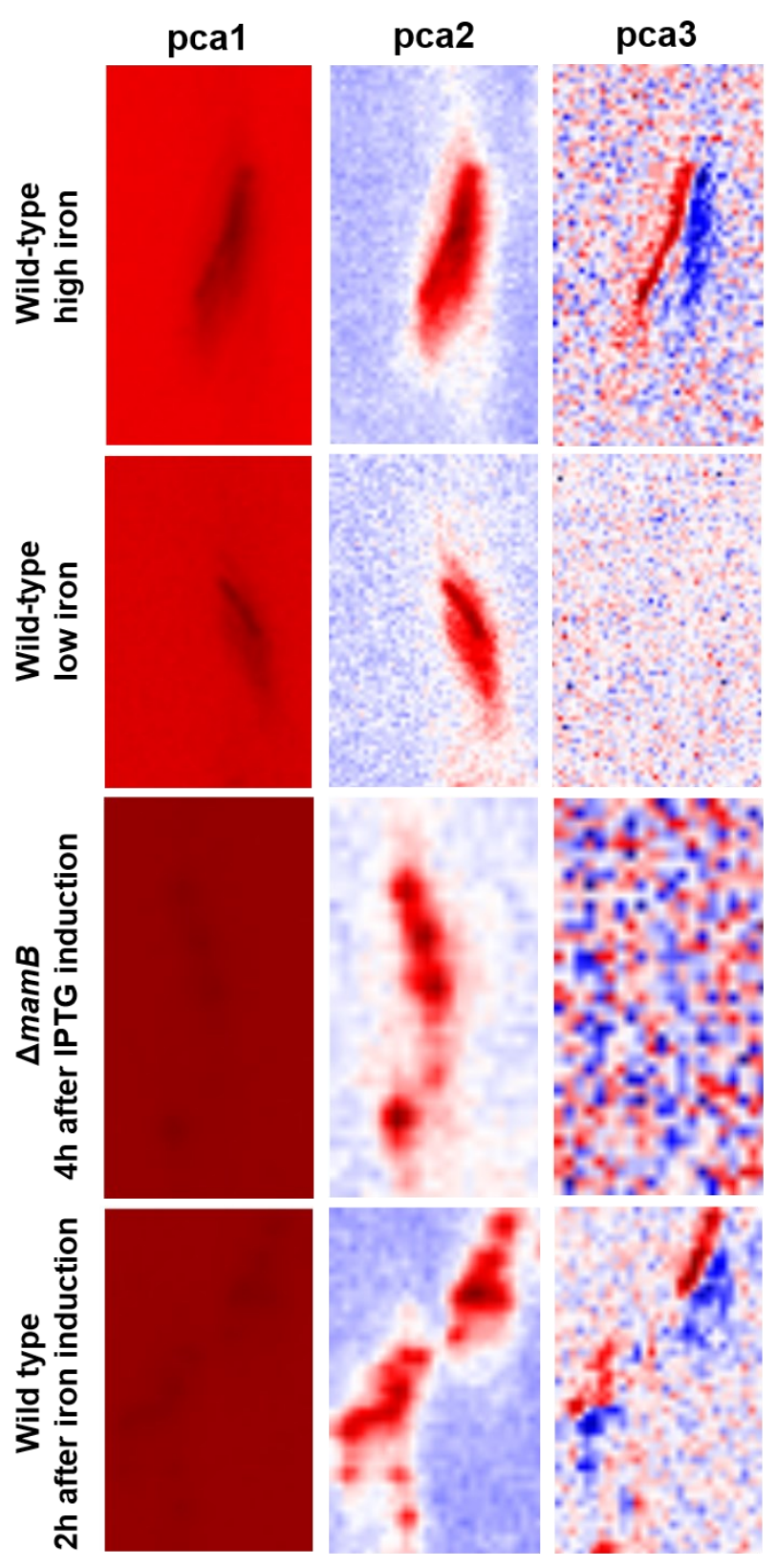

Fig S6. Principal components analysis maps for each sample. 

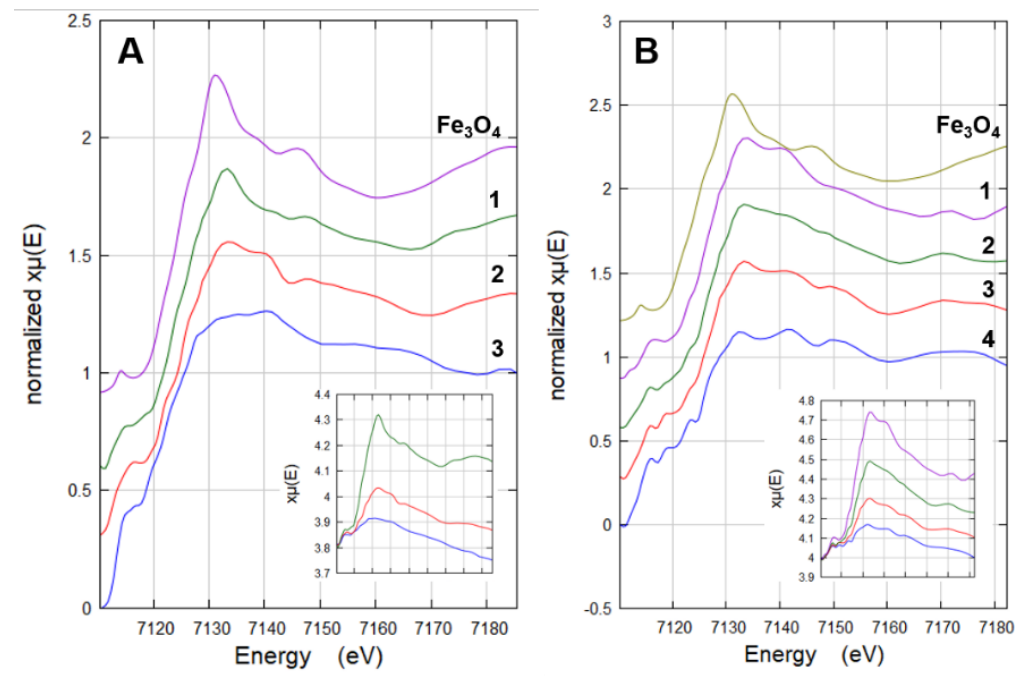

Fig S7. Fe K-edge XANES spectra for cluster centers with magnetite for (A) $\triangle m a m B$ high iron 4 $\mathrm{h}$ and (B) WT high iron $2 \mathrm{~h}$ (inset with spectra retaining edge jump values) 
Table S1. List of samples investigated and respective growth conditions and magnetosome induction method.

\begin{tabular}{|c|c|c|c|}
\hline Sample & $\begin{array}{l}50 \mu \mathrm{M} \mathrm{Fe}(\mathrm{III})- \\
\text { citrate added }\end{array}$ & $\begin{array}{c}\text { Time (h) of } \\
\text { extracted sample }\end{array}$ & $\begin{array}{c}\text { Magnetosome } \\
\text { formation induced }\end{array}$ \\
\hline WT high iron & Yes & 72 & No \\
\hline WT low iron & No & 72 & No \\
\hline$\triangle m a m B$ high iron $4 \mathrm{~h}$ & Yes & 4 & Yes (IPTG induction) \\
\hline WT high iron $2 \mathrm{~h}$ & Yes & 2 & Yes (Iron induction) \\
\hline
\end{tabular}


Table S2. Linear combination fitting results of Fe K-edge XANES spectra from full cell and cluster centers. $\Delta E_{0}=E_{\text {sample }, 0}-E_{\text {magnetite }, 0}$ relative oxidation from magnetite reference.

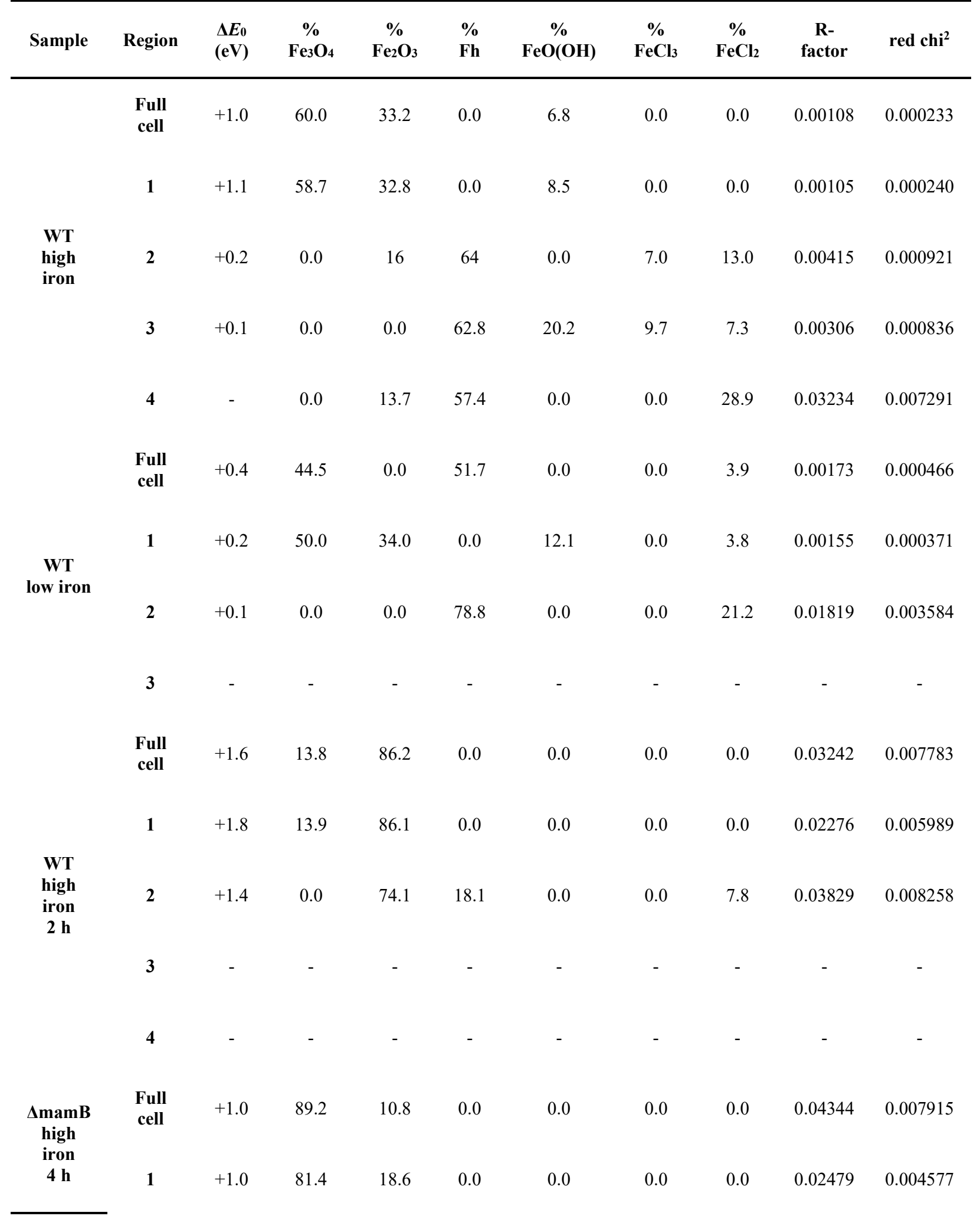


2

$\begin{array}{lll}+0.7 & 0.0 & 76\end{array}$

0.0

0.0

0.0

23.8

$0.05091 \quad 0.008981$

3 